\title{
Evidence of Precession of the White Dwarf in Cataclysmic Variables
}

\author{
Gaghik H. Tovmassian ${ }^{1,2}$ and Sergey V. Zharikov ${ }^{1}$ \\ Observatorio Astronómico Nacional, Instituto de Astronoma, UNAM, México. \\ \{gag, zhar\}@astrosen. unam.mx \\ and \\ Vitaly V. Neustroev \\ Computational Astrophysics Laboratory, National University of Ireland, Galway, Newcastle \\ $R d .$, Galway, Ireland. \\ Vitaly.Neustroev@nuigalway.ie
}

\begin{abstract}
Cataclysmic Variables (CV) are close binary systems, in which the primary, the more massive star, is a white dwarf. CVs usually exhibit a number of periodicities, most of which are now understood. However, recently, a new phenomenon was discovered that does not fit the standard picture. Two objects have been discovered to show periods that are much longer than orbital, and have no relation to it, either in light curves or in radial velocity (RV) variations measured from spectroscopy. Here, we show that the precession of the fast rotating magnetically accreting white dwarf can successfully explain these phenomena. The theory of compact objects predicts certain relations between the spin and precession periods, and our finding provides a good test for the theory and establishes a qualitative model to be explored both theoretically and observationally. Detection of precession can become a powerful tool in searching for the internal properties of compact stars, which would be otherwise inaccessible for us.
\end{abstract}

Subject headings: white dwarfs: precession, spin — cataclysmic variables: magnetic, individual(FS Aur, HS2331+3905)

\footnotetext{
${ }^{1}$ For correspondence use: PO Box 439027, San Diego, CA 92143-9027, USA.

${ }^{2}$ Visiting Research Fellow, CASS, UCSD
} 


\section{Introduction}

Cataclysmic Variables (CVs) are close binary systems, with typical orbital periods of a few hours. The more massive component in the binary, the primary star, is the white dwarf (WD) which accretes matter, usually through the disk formed around it. This produces number of periodic or quasi periodical signals in the light of the object observed both spectroscopically and photometrically (Warner 1995). Usually these signals are close to the orbital period (within 2-3\%), as in the case of superhumps, either positive or negative (Patterson 2001) or much shorter as in the case of Quasi-Periodic Oscillations (QPOs) and flickering, which are not periodic, but may introduce complicated patterns in the power spectra for the short time scales. In some cases, the final accretion occurs along the magnetic lines if the white dwarf is magnetized. In cases when the magnetic field of the primary exceeds $\approx 10 \mathrm{MG}$, the magnetosphere reaches beyond the disk radius, completely destroys it and locks the spin of the WD with the orbital period. Such objects are called Polars and they usually do not exhibit confusing periods. If the magnetic field of the WD is not so strong, but $\mathrm{B}>0.5 \mathrm{MG}$, the inner parts of the accretion disk become truncated, but the rest of the disk is not necessarily destroyed. However, the magnetic poles on the surface of the WD become a source of high energy beams due to the shock created by the accreting matter on them (similar to Polars). The beams functioning as a lighthouse allows detection of WD's strictly periodic spin period, which is not locked with the orbital period in this case. The spin period is normally shorter than the orbital period and often found to be about $10 \%$ of the latter. The beams from magnetic poles can irradiate other components of the binary system. Usually that creates a complex modulation of light observed from such objects, sub-classified as Intermediate Polars (IP). In spite of these complications, the period analysis of most IPs was a relatively easy task (Warner 1986; Norton et al. 1996).

Recently though, two objects were found to show periods that could not be explained within existing schemes. FS Aur, a seemingly ordinary dwarf nova system with an 85.7 min spectroscopic period was found to exhibit 3.425 hour photometric variations in its light curve (Tovmassian et al. 2003). Extended monitoring of the system over more than 10 years confirmed that the long photometric period has a variable amplitude and can be contaminated by other variability, but it remains stable and coherent (Neustroev et al. 2006). In order to explain the long photometric period in FS Aur, Tovmassian et al. (2003) invoked the IP model with a fast rotating and precessing, magnetically accreting white dwarf. Analyses of the fast ULTRACAM photometry of FS Aur suggests the presence of the quickly spinning white dwarf in the system (Neustroev et al. 2005).

HS2331+3905 was discovered later and immediately attracted attention for having two

unrelated spectral periods (Araujo-Betancor et al. 2004). Contrary to any conventional 
logic, it exhibits two very different spectral periods, a short one at the center, and a longer one in the wings of its emission lines. The radial velocity variations of the emission lines of CVs, thanks to the Doppler effect, are the principal means of period determination in binary stars. Luckily, HS2331+3905 is of high inclination and shows eclipses in its light curve, thus allowing an unambiguous determination of the orbital period of $81.08 \mathrm{~min}$. The second period ( $\sim 3.5$ hours), appearing in the far wings of the emission lines, has no explanation to date. Besides its two spectroscopic periods, HS2331+3905 exhibits a number of periodic photometric variations and is proved to contain a magnetic WD. The $67.2 \mathrm{sec}$ spin period of the WD defines this object as an IP (Araujo-Betancor et al. 2004).

This study is based on observations of these two CV systems that have unexplained long periods, one in its light curve and the other in radial velocities. Here, we report the detection of the second long (non-orbital) period in the RV measurements of emission lines of FS Aur that converges these two objects into a common case and suggests an universal picture explaining their behavior.

\section{Observations and reduction of data}

The observations of FS Aur were made at the $2.1 \mathrm{~m}$ telescope of San Pedro Martir Observatory in Mexico in December 2004. During two nights, using the Boller \& Chivens spectrograph, we gathered 81 spectra with total 12.4 hours of coverage of FS Aur with 420 sec individual exposure times. We obtained 54 spectra with FWHM $4.1 \AA$ resolution around the $\mathrm{H} \beta$ line and additional 27 spectra redward of $\mathrm{H} \alpha$. Including readout time between exposures, each spectrum covers about 0.1 orbital phase and approximately 0.03 phases of the long photometric period. The log of observations is presented in Table 1. Arc lamp exposures were obtained every 2 hours during long runs to insure proper wavelength calibration. For the flux calibration spectroscopic standard stars Feige67 and Feige110 were observed. The reduction was done using IRAF $^{1}$ routines which included bias subtraction, weighted extraction of the spectra, as well as wavelength and flux calibration.

The same telescope with the same instrument and slightly better resolution (FWHM $2.5 \AA$ ) were used during earlier observations of HS2331+3905 in September 2004 in a large

\footnotetext{
${ }^{1}$ IRAF is the Image Reduction and Analysis Facility, a general purpose software system for the reduction and analysis of astronomical data. IRAF is written and supported by the IRAF programming group at the National Optical Astronomy Observatories (NOAO) in Tucson, Arizona. NOAO is operated by the Association of Universities for Research in Astronomy (AURA), Inc., under cooperative agreement with the National Science Foundation
} 
multi-longitude, international campaign. The rest of our observations as well as a thorough analysis of larger set of data collected for this object will be presented in an upcoming paper by Gänsicke et al. (2006). In Table 1 there is an entry corresponding to the observation of HS2331+3905 used here. Actually we only use a trailed spectrogram of HS2331+3905 obtained by stacking about 70 spectra from 8 hours continuous exposures into one twodimensional image. The exposure times were also of $420 \mathrm{sec}$, similar to the data obtained for FS Aur. The spectra of both objects were normalized to the continuum before being stacked into two-dimensional trailed spectrum.

\section{The results}

\subsection{Evidence of the long period in the FS Aur spectra}

Usually FS Aur in its quiescence varies between aproximately $15 .^{m} 4-16 .{ }^{m} 2$ with an average magnitude about $15.7(\mathrm{~V})$ and shows a complex variability in the light curve which is a combination of the long term period $3.425 \mathrm{~h}$, the orbital period $85.7 \mathrm{~min}$ and QPO at short time scales (Neustroev et al. 2005; Tovmassian et al. 2003). The spectrum is typical for a dwarf nova with single-peaked strong and broad H, HeI emission lines and weak HeII $\lambda 4686$, CIII/NIII blend. The emission lines velocity variations clearly show the orbital period of the system reported by Thorstensen et al. (1996).

During the new spectral observations presented here, FS Aur was found to be more than half a magnitude fainter than expected for its quiescent state. It continued to decrease its brightness by about 1.5 magnitudes until the end of January 2005, after that coming back to the typical quiescent state. In Fig.1 a long term light curve is showing this trend. The $\mathrm{AAVSO}^{2}$ data demonstrate that the decrease in luminosity lasted about a few months. Such behavior is known in CVs as a VY Scl or anti-dwarf nova phenomenon. VY Scl objects are a rare group of CVs that undergo sudden and cyclical decrease of their luminosity, contrary to dwarf nova, that are called so, for showing cyclical outbursts. They are mostly concentrated in the 3-4 hour range of orbital periods, but recently shorter period systems have been found to exhibit the same kind of behavior (Rodriguez-Gil et al. 2004). Also in recent years there were attempts to link VY Scl phenomenon with truncation of the inner disk by the magnetosphere of the WD (Lasota \& Hameury 2004; Tovmassian 2005). The detection of a VY Scl type drop in brightness alone is an indication that FS Aur is probably a magnetic CV. The decreased brightness of the system, usually due to the drop in mass accretion rate

\footnotetext{
${ }^{2}$ www.aavso.org
} 
and mainly affecting the continuum level, allowed us to study faint wings of the lines with clarity.

At first glance, the new spectra of FS Aur looked similar to what was seen in previous observations, namely single peaked emission lines with a strong core whose sinusoidal RV variations define the orbital period. Generally it is thought that the center of the line originates at the outer edges of the accretion disk and is often dominated by a bright, hot spot at the point of impact of the stream of transfer matter with the disk. In order to measure radial velocities close to the WD, which will better reflect the orbital motion, the double Gaussian method (Schneider \& Young 1980) is used. The method allows for measurements of RVs in the far wings of the lines, originating in the inner parts of the accretion disk, close to the white dwarf. This technique involves convolving individual spectra with a double Gaussian template. When the counts in the two Gaussian bandpasses are equal, then the velocity of the emission line is assigned the value indicated by the midpoint between the two Gaussians. By varying the width and separation of the Gaussians, the velocity variations of various portions of the emission-line profiles is measured. This method is routinely used in study of CVs in order to measure extreme line wings in conditions of low $\mathrm{S} / \mathrm{N}$ and determine radial velocity of the primary.

Usually in CVs, the line wings at different Gaussian separations show sinusoidal curves of the same period (orbital), sometimes displaced in phases relative to each other. The pattern that emerged in case of new observations of FS Aur was completely different. Using the standard Lomb-Scargle power spectrum analysis (Lomb 1976; Scargle 1982), we calculated the power spectra of the RV measurements with four distinct separations of the double Gaussians (Fig. 2). At $600 \mathrm{~km} / \mathrm{sec}$ separation, there is the power peak at the frequency corresponding to the orbital period. As the separation increases, the peak corresponding to the orbital period decreases in strength, while a second strong peak emerges. Frequency of this peak is equal exactly to the beat between the orbital and $3.425 \mathrm{~h}$ photometric periods: $1 /$ Pbeat=1/Porb $-1 /$ Pphot. By separation of $2000 \mathrm{~km} / \mathrm{sec}$, all power is concentrated in the beat period. The significance levels of the power peaks in Fig 2 far exceed $99.99 \%$ and the result is repeated with other emission lines $(\mathrm{H} \gamma$ and $\mathrm{H} \alpha)$, thus leaving no doubt as to its reality. It must be noted that a peak at the beat $\left(1 / P_{\text {orb }}-1 / P_{\text {phot }}\right)$ period was detected in our earlier observations of FS Aur (Tovmassian et al. 2003), which is another indication of real and repetitive nature of the discovered phenomenon. Back then, the object was brighter, which means that the accretion disk was brighter, and the modulation in the wings of the line with different period was not so apparent. The new observations of FS Aur in a low luminosity state permit detecting the produced effect by naked eye. We constructed a trailed spectrum (two dimensional image of the emission line where the x-axes are wavelengths or radial velocities and the $y$-axes are times) presented in Fig. 3. The intense central part of the 
line varies reflecting the orbital motion of the system, but the wings of the line show waves with a different period, i.e. the beat period between the orbital and $3.425 \mathrm{~h}$ photometric periods. On the flanks of the trailed spectra we plotted measurements of radial velocities of corresponding portions of wings of the line. The RV curve measured near the center of the line is presented on the left and measurements in the extreme wings are in the utmost right panel. The radial velocity curves are even more demonstrative when folded with the corresponding periods. In Fig 4 sets of panels are presented. On the left side the RV measured at $600 \mathrm{~km} / \mathrm{sec}$ Gaussian separation or within $\pm 300 \mathrm{~km} / \mathrm{sec}$ from the center are folded with the orbital period on top and $147 \mathrm{~min}$ beat period on the bottom. On the right side the RV measurements in far wings of the line at $1600 \mathrm{~km} / \mathrm{sec}$ separation are folded with the same periods. As one can see, the center of the line forms the expected sinusoid when folded with the orbital period, while the wings of the line fold correctly only with the long period. When one dataset is folded with the period derived from the second dataset, then RV curve becomes confused - and vice versa. In this Figure we also plotted measurements of $\mathrm{H} \gamma$ line to show that pattern repeats from line to line.

The wings of the emission lines in FS Aur are considerably fainter than those in HS2331+3905, in which the double period can be seen clearly on the trailed spectrogram (see Fig. 6, left panel). This unusual pattern was first reported by Araujo-Betancor et al. (2005) and persists in later observations. Until now it had been an unique phenomenon reported without any explanation. In Fig. 6, we present a sample from our observations of HS2331+3905, which vividly demonstrates this peculiar phenomenon and, as such, is better suited for a comparison with the model which we developed.

HS2331 +3905 is an eclipsing CV, therefore its inclination is much higher $\left(i>75^{\circ}\right)$ than of FS Aur for which no exact estimates of inclination angle exists, but it is believed to be in range of $50^{\circ}<i<65^{\circ}$ (Neustroev 2002; Tovmassian et al. 2003). The difference in inclinations probably can explain why the wings of HS2331+3905 demonstrate a spectacular effect, while for FS Aur it is barely seen. However, a more important reason here can be the difference in mass-transfer rates in these systems. A number of arguments suggest that HS2331+3905 is a CV with a very low mass transfer rate (Araujo-Betancor et al. 2005), that means the accretion disk in this system is optically thin and slim. On the contrary, the mass transfer rate in FS Aur is quite high $\left(\sim 4 \times 10^{10} M_{\odot} / y r-\right.$ Urban \& Sion 2006), thus its accretion disk is expected to be thick both optically and geometrically. Such disk can envelope the WD and obscure central parts, which only can be seen occasionally through the scattered radiation in the polar directions of the disk. A decrease of the mass transfer rate could uncover the innermost disk and allow us to see this area more clearly. Of course, differences in magnetic field strength and higher spectral resolution during observations of HS2331+3905 also contribute to the appearance of the emission lines. 


\subsection{Common case and the model}

The evidence for the second period in the wings of FS Aur emission lines, directly related to the long photometric period, provided the crucial link between itself and HS2331+3905. The parameters of both objects, summarised in Tab. 2, are also very similar. Whilst before, one only could speculate where the long photometric period in FS Aur was originating from, now it is without doubt clear that it is associated with the internal parts of the accretion disk.

The two objects suggest universal picture, whose common feature, we believe, is a fast rotating and precessing, magnetically accreting white dwarf. We built a simple, basically qualitative model of the accretion disk that recreates the observed features of the trailed spectrogram of these objects, in particular of HS2331+3905. The basic characteristics of the model are:

1. The system should be moderately magnetic (IP) in order to create conditions where the accretion disk exists, but is truncated. More importantly we need an accreting magnetic pole on the white dwarf that will alter the structure of the inner disk either by irradiation or geometrically (e.g. warp, density bulge, etc)

2. Rotation of the spot around the inner radius of the disk in sync with the precessing WD.

In Fig 5 we present a schematic drawing of the proposed model. In order to simulate emission line profiles formed by the accretion disk of HS2331+3905, we have applied a threecomponent model that includes a geometrically thin Keplerian accretion disk and two bright spots. The first spot, on the outer rim of the accretion disk, maintains a constant position with respect to components of the binary system, while the second spot moves around the inner edge of the disk to follow the precession of the WD. We began the modeling of the line profiles with calculation of a symmetrical double-peaked profile formed in the uniform axisymmetrical disk, then added the distorting components formed in the bright spots. To calculate the emission line profiles we divide the disk surface into a grid of elements and assign the velocity vector, line strength and other parameters for each element. The computation of the profiles proceeds by summing the local line profiles weighted by the areas of the surface elements, taking into account the Keplerian velocity gradient across the finite thickness of the disk (Horne \& Marsh 1986). We have assumed a power law function for distribution of the local line emissivity $f(r)$ over the disk surface $f(r) \sim r^{-\alpha}$, where $r$ is the radial distance from the disk centre and $\alpha=1-2.5$ (Smak 1981; Horne \& Saar 1991). For calculation of the spot's emission components we consider the spots on the accretion disk 
to have a quasi-rectangular shape and a Keplerian velocity. The local line emissivity $f(r)$ over the spots areas was simply increased by some constant value with comparison to the underlying accretion disk. For details of the method see Horne \& Marsh (1986) and Horne (1995), whereas its extension for a non-uniform accretion disk is described by Neustroev et al. (2002). On the base of this model we calculated a time dependent sequence of emission line profiles. The location of the spots, at any given time, was determined by their current orbital and precession phase. The first is at the usual place on the outer rim which is common for most CVs co-rotating with the binary, while the other is on the inner edge of the disk and whose location is determined by interaction with the precessing WD. The corresponding trailed spectrogram produced by this model is presented in Fig 6, right panel.

The inner disk should be warped by the interaction between the magnetosphere and the disk, but the heating mechanism is not clear at this point. Theory predicts a higher density in parts of the disk closest to the magnetic pole (Romanova et al. 2004). The warped disk as well as the higher density spot will move and change its angle toward an observer following the precession axis. There is also the possibility of the irradiation of parts of the inner disk by the accretion spot on the white dwarf. The irradiation phenomenon is detected routinely in Polars, where there is no disk, and the beam from the accretion spot irradiates the facing side of the secondary star (Warner 1995). As already mentioned the model is qualitative (or rather geometrical) and does not address the physical processes responsible for heating or emmisivity of spots or the disk in general.

\subsection{Why precession of WD and why these two objects?}

With the assumption that there is a bright spot at the inner side of the accretion disk, we were able to mimic the behavior of the emission lines of these two objects. But the model is incomplete without a mechanism that makes the inner spot move around in a periodic manner, independent of the orbital period. In Tovmassian et al. (2003) we discussed a variety of scenarios and suggested that the precession of the white dwarf was the possible solution, provided that the white dwarf in FS Aur is accreting magnetically and rapidly rotating. The argument was based on a simple premise that such precession would provide a period comparable to the observed one. According to Leins et al. (1992), the Euler frequency of free precession $\omega_{e}$ of a rigid and axially symmetric body or the Chandler frequency $\omega_{c}$ of an elastic body will be:

$$
\omega_{e, c}=\alpha_{e, c}\left(\rho_{c}\right) \Omega^{3}
$$

where $\alpha_{e, c}$ are coefficients that are determined by the central density $\rho_{c}$ and $\Omega$ is the rotation frequency of a WD. As a result a WD with spin period in range of 50 to 100 sec should 
have a precession period of a few hours ${ }^{3}$, depending on mass of the WD. Periods observed in HS2331+3905 satisfy spin/precession ratio and strengthen our claim that precession of the WD is responsible for observed phenomena. This also gives more credibility to the $100 \mathrm{sec}$ period found in the power spectrum of FS Aur in the optical (Neustroev et al. 2005) and X-rays (Tovmassian et al. 2006).

In Tovmassian et al. (2003) we also speculated that the discovered long photometric period of FS Aur was the precession period of the WD. However now, when we found the spectroscopic period at the beat frequency between orbital and photometric, and compare two objects, we should revise the assignment. It seems natural that the spectroscopic period will be a direct measure of rotation of the inner spot (precession period according to our hypothesis) and that the period seen in photometry will be the beat. Indeed, there is no way to produce a significant radial velocity signal on the beat period (at least we were unable to do), but there is no problem to do this for photometric variations. Following a similar strategy to that of Warner (1986), one can see that any structures which are co-rotating with the binary with frequency $\omega_{\text {orb }}$ and which are periodically illuminated by the beam as it precesses around the system with frequency $\omega_{\text {prec }}$, will vary in brightness with frequency $\left(\omega_{\text {orb }}-\omega_{\text {prec }}\right)=\omega_{\text {phot }}$.

Considering the long spectroscopic period as the precession, we still reach agreement between the spin and precession periods for FS Aur according to (1). In Fig 7 we present the relationship of the precession period to the spin period, as follows from the Leins et al. (1992) analysis. The solid lines are ratios determined by using the Euler frequency for a WD with masses indicated by the numbers at the top of the figure. Corresponding Chandler dependences are shown with dashed lines within the range of masses indicated lower. The location of FS Aur and HS2331+3905 are marked by filled symbols, which assumes that the precession period corresponds to spectroscopic period. If the photometric period is the period of precession then the symbols shift to the locations marked by the open symbols. In both cases the masses of the WDs in both systems make perfect sense, given that they act as rigid bodies and the Euler frequencies are applicable. In plotting this figure we calculated what the long photometric period of HS2331+3905 would be as a beat period between observed spin and long spectroscopic periods, but it has not been detected.

Assuming that the mean white dwarf mass in $\mathrm{CVs}$ is of about $0.6 \mathrm{M}_{\odot}$, only those WDs whose spin period is $\leq 120 \mathrm{sec}$ would have a precession period of less than 8 hours that could

\footnotetext{
${ }^{3}$ Independently, Schwarzenberg-Czerny (1992) made rough estimates of $P_{\text {prec }} / P_{\text {spin }} \sim 10^{4}-10^{5}$ using slow rotating polytropic star models and obtained results similar to Leins et al. (1992) for long spin periods typical of majority of IPs, but his calculations were not intended for rapid rotators.
} 
be detectable in a single night's observing. Detection of longer periods is not impossible of course, and is a complicated task and we are not sure anyone ever looked at it. The number of systems that are proven to be IP with established spin periods is very limitted. One other known rapid rotator is DQ Her. There are no reports of additional long periods detected in this system, but its orbital period is 4.65 hours (quite different from objects discussed here and compatible with the hypothetical precession period) and it has a huge accretion disk (Wood et al. 2005). Again, we would like to emphasize, that when FS Aur is observed in normal quiescence, the long period is barely detectable in the wings of the lines. So, while the free precession of white dwarfs in CVs can be a common phenomenon it is not something that is easily detectable.

\section{Conclusions}

We demonstrate that the two enigmatic Cataclysmic Variables FS Aur and HS 2331+3905, which were known to show periods much longer than their orbital period in the photometric light curve and the wings of the emission lines, respectively, have the same nature. The clarification derives from the discovery of the second period in the wings of the accretion lines of FS Aur, the same phenomenon that was first seen in HS2331+3905. This allows us to consider them both as Intermediate Polars, which, in the case of HS2331+3905, is an observationally proven fact. If this is true, then the model of a fast rotating and precessing, magnetically accreting white dwarf proposed earlier for FS Aur, works for both systems and allows for the explanation of outstanding features observed in these systems, as demonstrated by the model of an accretion disk with a bright spot on the inner rim following the precession axis. This hypothetical inner bright spot is not well defined, it could be the irradiated inner edge of the disk, condensation of matter in it or a warped inner disk which are all due to interaction with the magnetosphere. The differences in appearance of the long photometric (beat) period (there was no such period reported for HS2331+3905) and the strength of the modulation in the wings of the emission lines in the two systems can be accounted for by the difference in inclination angles and/or mass-transfer rates.

If our analysis is correct, then this will be the first instance when the precession of the WD is detected in a more or less straightforward manner. Combined with other methods to estimate masses and density of the WDs in CVs, the precession period will help to refine these values, which in turn, allows for testing and proving the theory for the precession of compact stars and may allow for further advances in our knowledge of the structure of WDs and the influence of accretion on them. 
We acknowledge CONACyT grant 45847. VN acknowledges support of IRCSET under their basic research programme and the support of the HEA funded CosmoGrid project. We appreciate discussion and comments made by Dr. M. Romanova regarding the magnetic accretion. We also acknowledge useful discussion of the subject with Dr. B. Gansicke. We are thankful to Drs. M. Richer \& J.Tomsick for careful reading of the manuscript.

\section{REFERENCES}

Araujo-Betancor S., GNsicke, B. T., Hagen, H.-J., Marsh, T.R., Thorstensen, J., Harlaftis, E.T., Fried, R.E., Engelset, D., 2004, Revista Mexicana De Astronoma Y Astrofsica (Serie De Conferencias) 20, 190

Araujo-Betancor, S., Gänsicke, B.T. , Hagen, H.-J., Marsh, T.R., Harlaftis, E.T., Thorstensen, J., Fried, R.F., Schmeer, P., Engels, D., 2005, A\&A, 430, 629

Gänsicke B., et al. 2006, in preparation

Horne, K., Marsh, T.R., 1986, MNRAS218, 761

Horne, K., Saar, S.H., 1991, ApJ, 374, L55

Horne K., 1995, A\&A, 297, 273

Lasota, J.-P., Hameury, J.-M. 2004, Magnetic Cataclysmic Variables, IAU Colloquium 190, ASP Conference Proceedings 315, 46

Leins, M., Soffel, M.H., Lay, W., Ruder, H., 1992, A\&A. 261, 658

Lomb N. R., 1976, Ap\&SS, 39, 447

Neustroev, V. V. 2002, A\&A, 382, 974

Neustroev V.V., Borisov N.V., Barwig H., Bobinger A., Mantel K.H., Simic D., Wolf S., 2002, A\&A, 393, 239

Neustroev, V.V., Zharikov, S.V., Tovmassian, G., Shearer, A., 2005, MNRAS, 362, 1472

Neustroev V.V., et al., 2006 in preparation

Norton, A. J., Beardmore, A. P., \& Taylor, P. 1996, MNRAS, 280, 937

Patterson, J. 2001, PASP, 113, 736 
Rodrguez-Gil, P., Gänsicke, B. T., Araujo-Betancor, S., 2004, MNRAS, 349, 367

Romanova, M.M., Ustyugova, G.V., Koldoba, A.V., Lovelace, R.V.E., 2004, ApJ, 610, 920

Scargle J.D., 1982, ApJ, 263, 835

Schneider, D.P., Young, P., 1980, ApJ, 238, 946

Smak J., 1981, AcA, 31, 395

Schwarzenberg-Czerny, A. 1992, A\&A, 260, 268

Thorstensen, J.R., Patterson, J.O., Shambrook, A., Thomas, G. 1996, PASP, 108, 73

Tovmassian, G., Zharikov, S., Michel, R., Neustroev, V., Greiner, J., Skillman, D. R., Harvey, D. A., Fried, R. E., Patterson, J., 2003, PASP, 115, 725

Tovmassian, G., 2005, Interacting Binaries: Accretion, Evolution, and Outcomes. AIP Conference Proceedings, 797, 257

Tovmassian, G. et al., (2006) in preparation

Urban, J. A., \& Sion, E. M. 2006, ApJ, 642, 1029

Warner, B. 1986, MNRAS, 219, 347

Warner, B., 1995 in Cataclysmic Variable Stars (Cambridge:Cambridge Univ. Press)

Wood, M. A., et al. 2005, ApJ, 634, 570 
Table 1: Log of observations

\begin{tabular}{|c|c|c|c|c|}
\hline $\begin{array}{l}\text { Object } \\
\text { Spectroscopy }\end{array}$ & Date & $\begin{array}{l}\text { HJD+ } \\
2450000\end{array}$ & Instrument/Grating $^{a} /$ Range/Exp.Time $\times \mathrm{N}^{b}$ & Duration \\
\hline FS Aur & $2004-12-08$ & 3347 & $4001 / \mathrm{mm} 3500-5500 \AA 420 \mathrm{~s} \times 37$ & $5.2 \mathrm{~h}$ \\
\hline FS Aur & 2004-12-08 & 3347 & $4001 / \mathrm{mm} 6470-8500 \AA 420 \mathrm{~s} \times 17$ & $2.4 \mathrm{~h}$ \\
\hline FS Aur & 2004-12-09 & 3348 & $400 \mathrm{l} / \mathrm{mm} 3500-5500 \AA 420 \mathrm{~s} \times 27$ & $3.7 \mathrm{~h}$ \\
\hline HS2331+3905 & 2004-09-15 & 3263 & $1200 \mathrm{l} / \mathrm{mm} 4340-5900 \AA 420 \mathrm{~s} \times 67$ & $8.6 \mathrm{~h}$ \\
\hline
\end{tabular}

${ }^{a}$ - Boller \& Chivens spectrograph (http://haro.astrospp.unam.mx/Instruments/)

${ }^{b}$ - Number of Integration 
Table 2: Parameters of Binary Systems

\begin{tabular}{lcc}
\hline \hline & FS Aur & HS 2331+3905 \\
\hline Orbital Period & $85.7 \mathrm{~min}^{1}$ & $81.08 \mathrm{~min}$ \\
Spin Period & $\sim 100 \mathrm{sec}^{3}$ & $67.2 \mathrm{sec}$ \\
Long photometric period & $205.5 \mathrm{~min}^{4}$ & - \\
Long spectroscopic period & $147 \mathrm{~min}^{5}$ & $196.5 \mathrm{~min}{ }^{5}$ \\
Inclination angle & $\left(50^{\circ}-65^{\circ}\right)^{6}$ & $>75^{\circ}$ \\
\hline
\end{tabular}

1 - Thorstensen et al. (1996), 2 - Araujo-Betancor et al. (2004),

3 - Neustroev et al. (2005), 4 - Tovmassian et al. (2003),

5 - this paper, 6 - Neustroev (2002) 


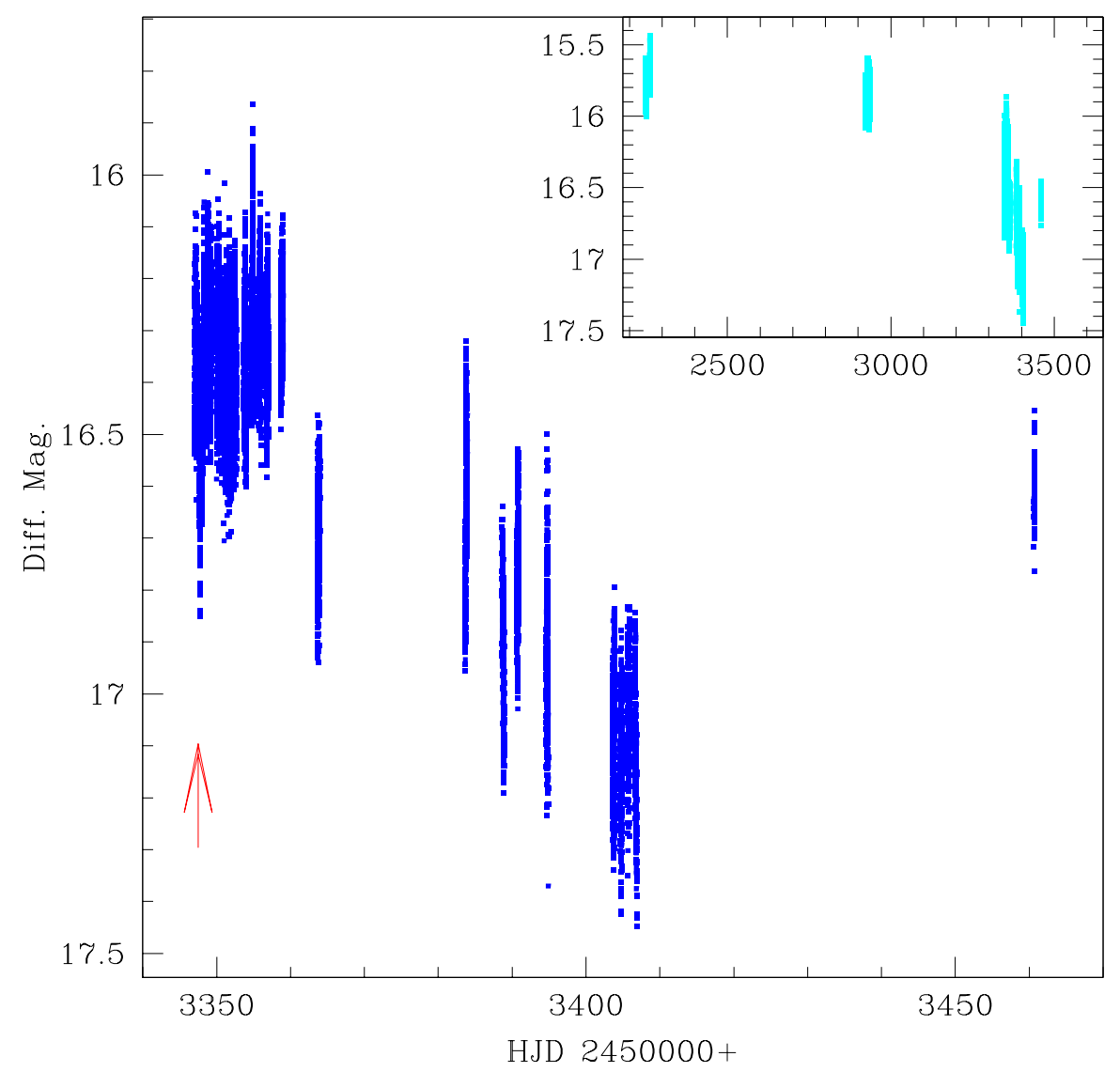

Fig. 1. - The light curve of FS Aur. The spectroscopic observations reported in this paper were performed during two nights marked with the arrow. The normal quiescence brightness of FS Aur is $\approx 15.7(\mathrm{~V}))$. The inset of the figure shows long term light curve prior to our new observations where the object was in quiescence. During the spectroscopy the object was already in a low state and shows an anti-dwarf nova drop in brightness afterwards. 


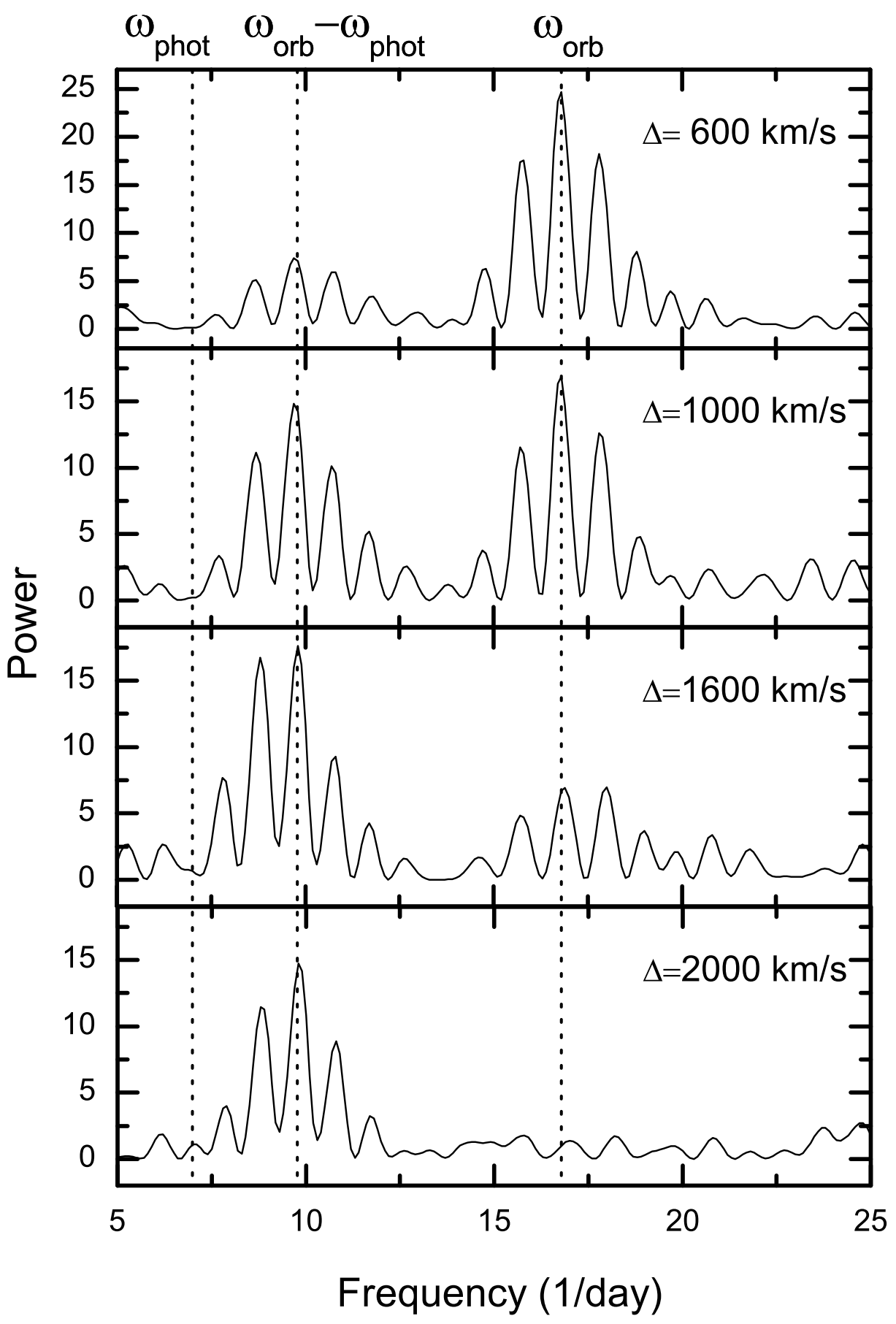

Fig. 2.- The periodogram of radial velocity variations of the $\mathrm{H} \beta$ line of FS Aur, measured by the double Gaussian method. There were 81 spectra measured, spanning 11.4 hours on two consecutive nights ( 4 photometric periods). In the upper panel, the period calculations (Scargle 1982) are based on measurements with the $600 \mathrm{~km} / \mathrm{sec}$ separation of the Gaussians, and the power in the spectrum peaks at the orbital period. Increasing the separation to 1000 $\mathrm{km} / \mathrm{sec}$ produces the emergence of the second significant period. At $1600 \mathrm{~km} / \mathrm{sec}$ the second period becomes dominant and coincides exactly with the beat period between orbital and photometric periods observed in FS Aur. 

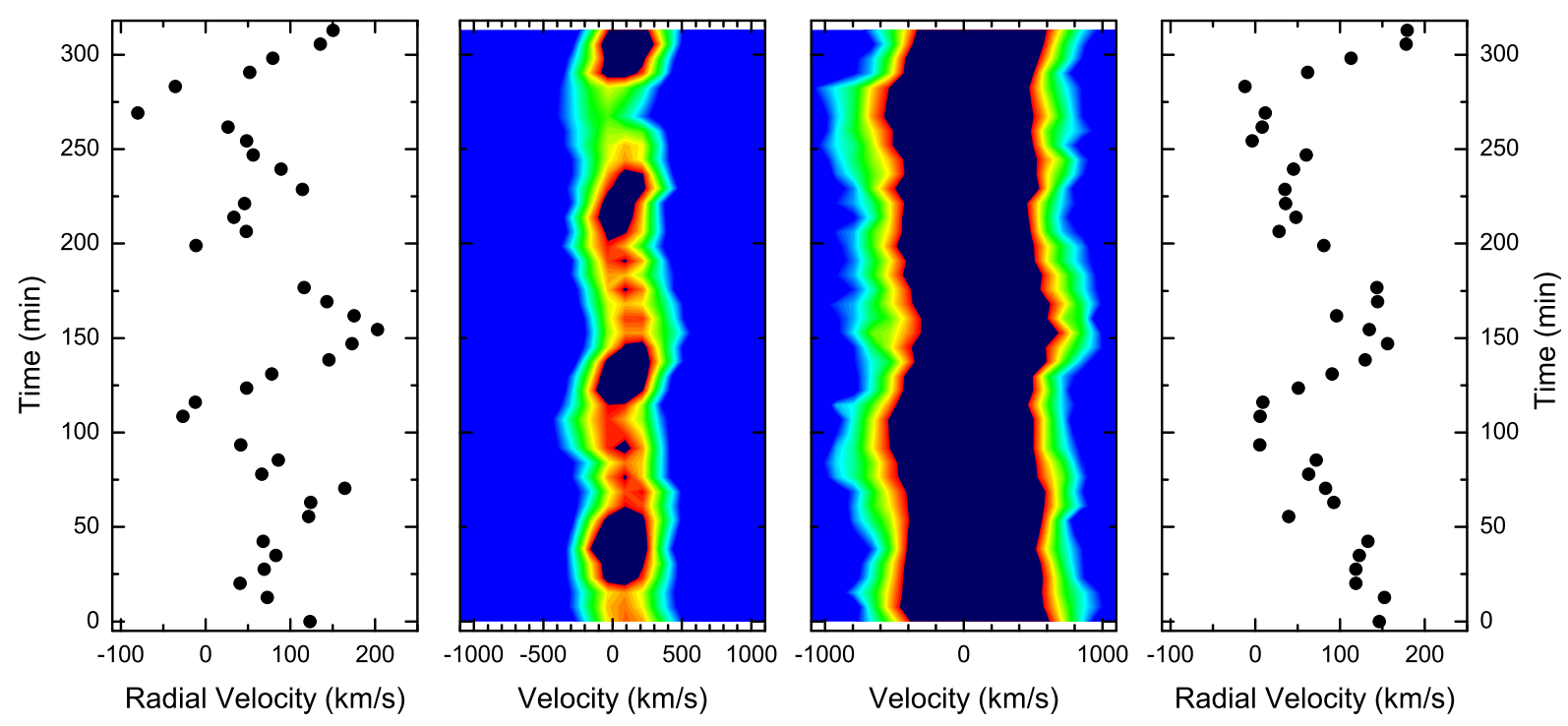

Fig. 3. - The trailed spectrogram of $\mathrm{H} \beta$ emission line of FS Aur made of 37 individual spectra obtained during the first observing night, are shown on two central panels with different contrast to emphasis different components of the line. The trailed spectra are flanked by their corresponding RV curves. The first panel on the left presents measurements of the center of the line with double Gaussian separation of $600 \mathrm{~km} / \mathrm{sec}$. The last panel presents measurements with $1600 \mathrm{~km} / \mathrm{sec}$ separation. The emission line is modulated with two periods. The central part varies according to the orbital period, while wings of the line better seen in the right panels are modulated with the beat period (147 $\mathrm{min}$ ) between the orbital and long photometric period. 

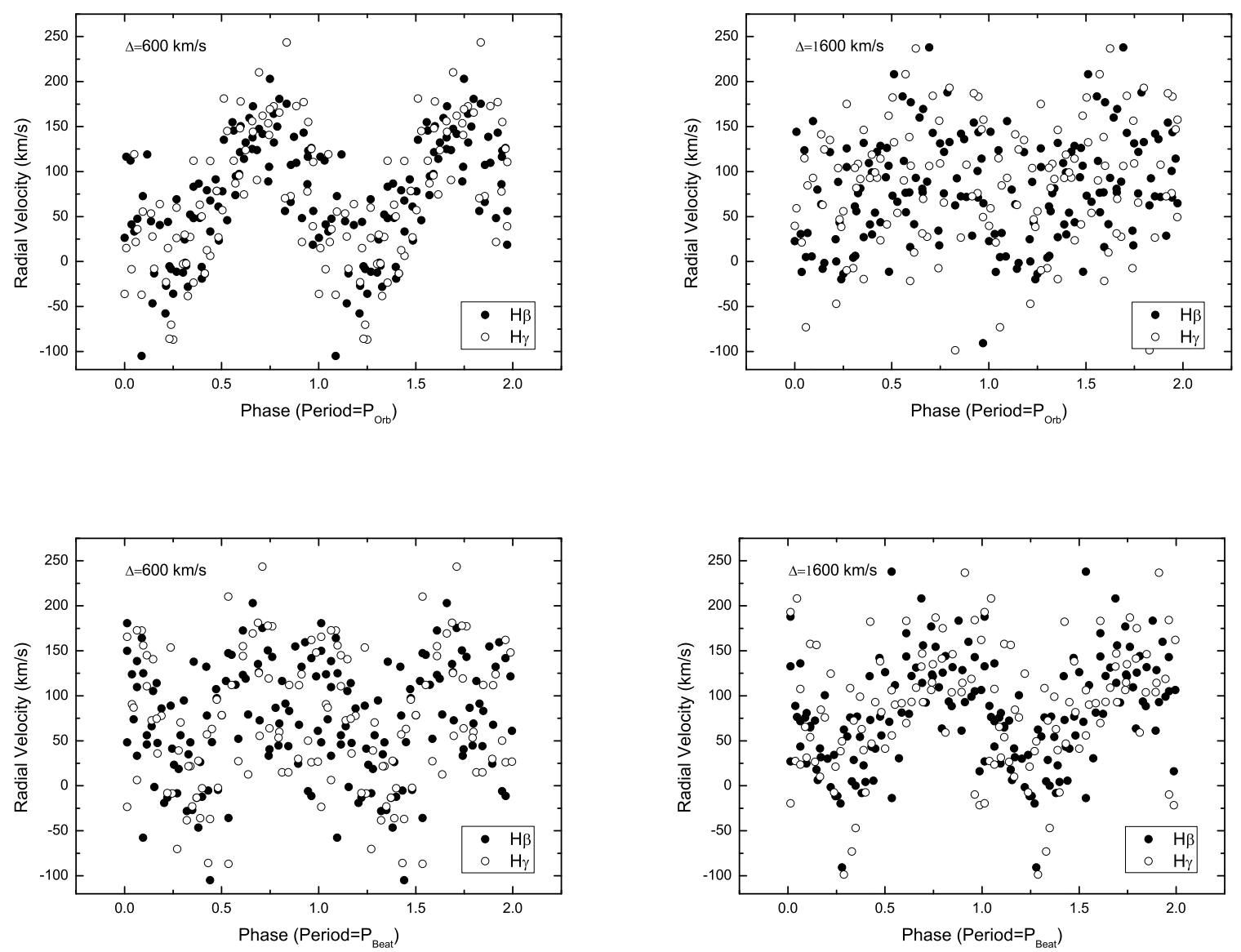

Fig. 4. - The radial velocity curves. On the top left: RV measurements of $\mathrm{H} \beta$ and $\mathrm{H} \gamma$ lines with double gaussian separation of $600 \mathrm{~km} / \mathrm{sec}$ folded with the orbital period of FS Aur. On the left bottom are the same measurements folded with the long period. On the left side corresponding curves plotted using the RV measurements with the gaussian separation of $1600 \mathrm{~km} / \mathrm{sec}$, corresponding to $800 \mathrm{~km} / \mathrm{sec}$ velocity in the emission line wings. 


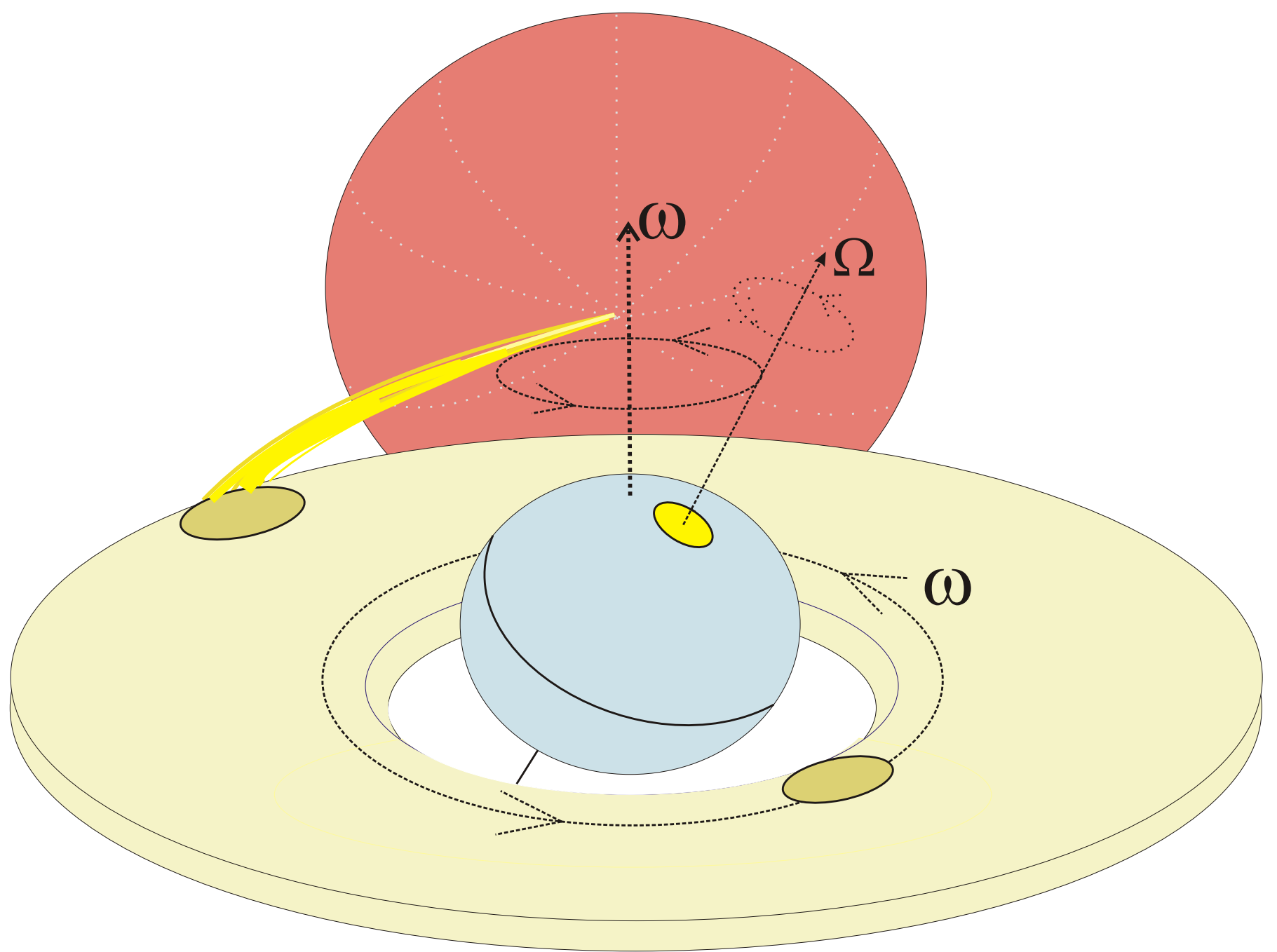

Fig. 5.- A schematic model of a FS Aur type CV with a rapidly rotating and precessing magnetic white dwarf as a primary, and a truncated accretion disc. The disc has a heated bright spot inside, which is not stationary, but follows the rotation axis of the WD as it slowly precesses. The origin of heating is not clear at this moment, but can be explained by variety of reasons. There is another bright, hot spot on the outer edge of the disc, which is stationary and located where the stream of mass transfer impacts the disc. 

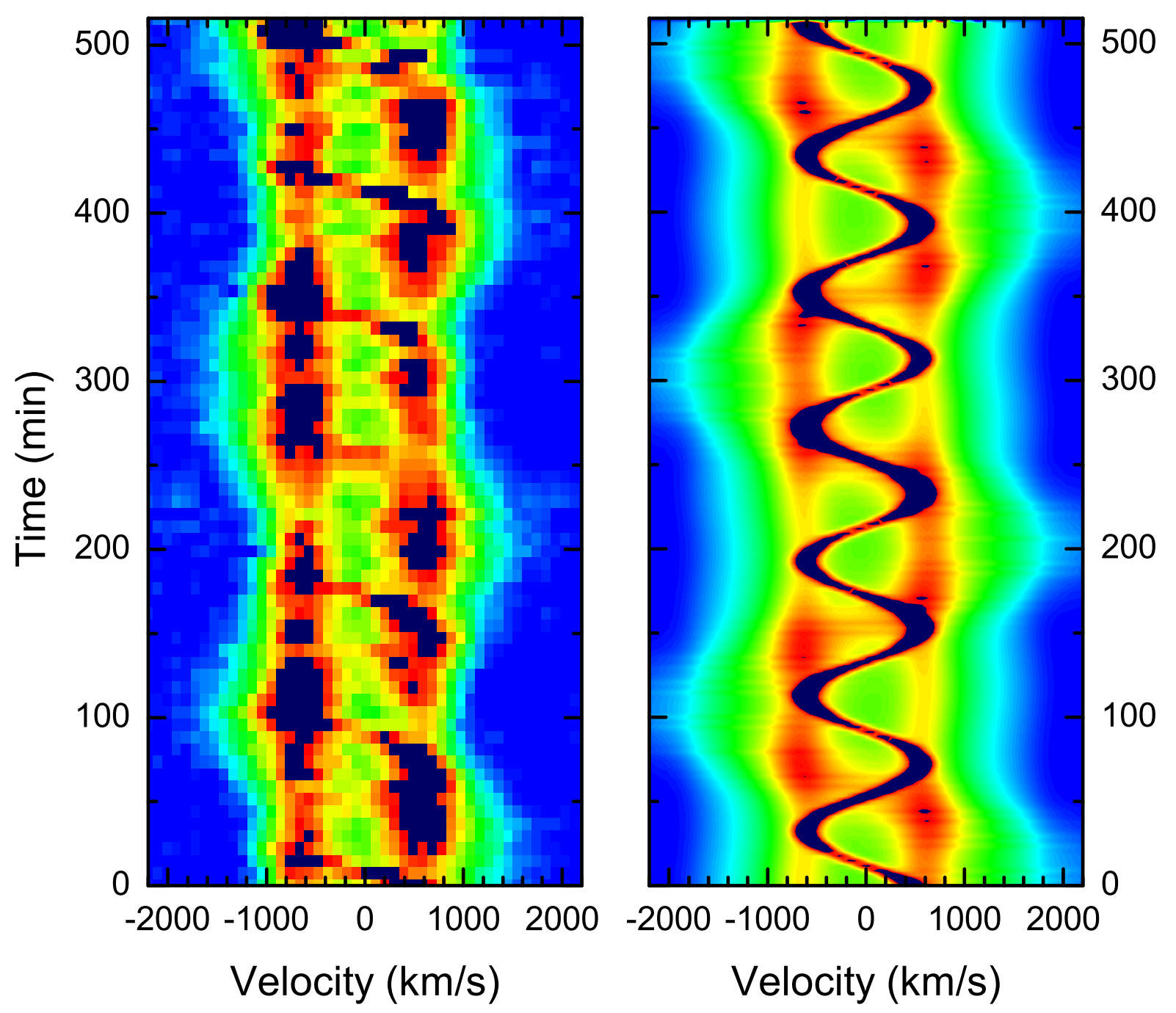

Fig. 6. - Trailed spectrogram of HS2331+3905 H $\beta$ emission line is on the left panel. The spectra were obtained at the OAN SPM 2.1-m telescope during a multi-longitude campaign in 2004. The details of the observations and results will appear in Gänsicke et al. (2006). The model describing CV system containing precessing, magnetic and rapidly rotating WD is on the right panel. We produced this image based upon a three-component model that includes a geometrically thin Keplerian accretion disk and two bright spots. 


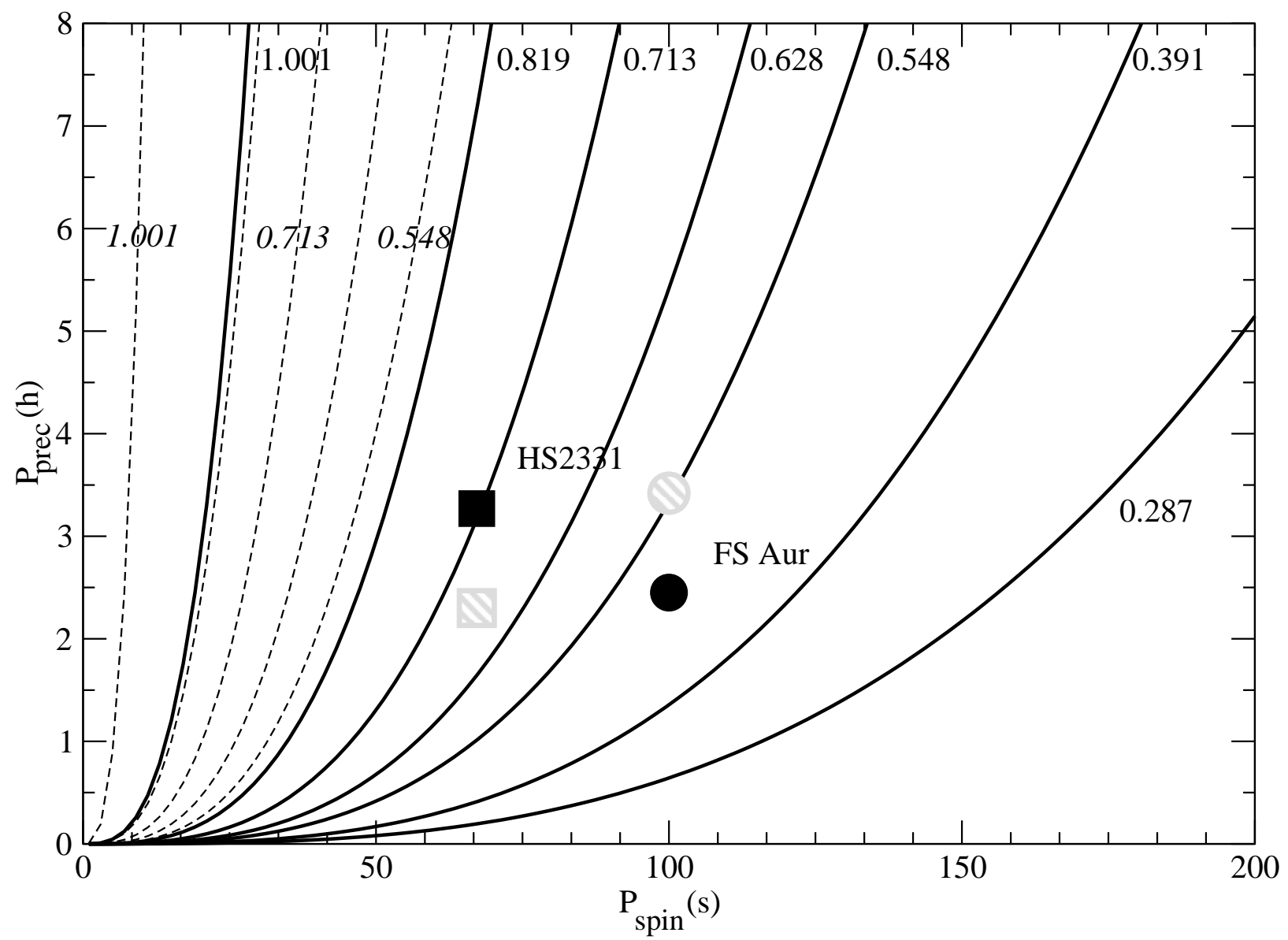

Fig. 7.- The relation $P_{\text {prec }}$ vs. $P_{\text {spin }}$ for WDs with different masses in case of Euler (solid lines) and Chandler frequency (dashed lines) from Leins et al., (1992). Numbers mark the mass of WD for each curve. Filled circle and square denote long spectral periods of FS Aur and HS2331+3905 respectively. Hashed circle mark long photometric period of FS Aur, which corresponds to the beat period between orbital and long spectroscopic periods. Respective beat period for HS2331+3905, marked by hashed square is actually not detected. We assume that spectroscopic, rather than photometric, period corresponds to the precession period, thus filled symbols should indicate correct positions of the systems in the plot. 